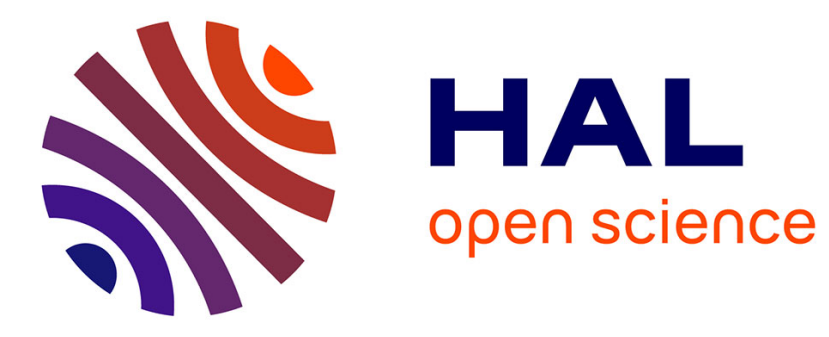

\title{
Christian Bromberger, Le match de football
}

Dominique Marchetti

\section{To cite this version:}

Dominique Marchetti. Christian Bromberger, Le match de football. Politix, 1996, p. 258-261. 10.3406/polix.1996.1968 . halshs-02379791

\section{HAL Id: halshs-02379791 https://shs.hal.science/halshs-02379791}

Submitted on 25 Nov 2019

HAL is a multi-disciplinary open access archive for the deposit and dissemination of scientific research documents, whether they are published or not. The documents may come from teaching and research institutions in France or abroad, or from public or private research centers.
L'archive ouverte pluridisciplinaire HAL, est destinée au dépôt et à la diffusion de documents scientifiques de niveau recherche, publiés ou non, émanant des établissements d'enseignement et de recherche français ou étrangers, des laboratoires publics ou privés. 


\section{Bromberger, Le match de football}

In: Politix. Vol. 9, №35. Troisième trimestre 1996. pp. 258-261.

Citer ce document / Cite this document :

Marchetti Dominique. C. Bromberger, Le match de football. In: Politix. Vol. 9, №35. Troisième trimestre 1996. pp. $258-261$.

http://www.persee.fr/web/revues/home/prescript/article/polix_0295-2319_1996_num_9_35_1968 
BROMBERGER (Christian) avec la collaboration de HAYOT (Alain) et MARIOTTINI (Jean-Marc), Le match de football. Ethnologie d'une passion partisane a Marsetlle, Naples et Turin, Paris, Éditions de la Maison des sciences de l'homme, 1995, 406 pages, index.

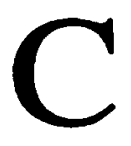

OMMENT expliquer l'engouement que suscite le football ? C'est à cette question que tente de répondre Christian Bromberger, ethnologue à l'université de Provence, dans un des rares ouvrages consacrés aux supporters des matches de football. Son livre est d'abord une nouvelle et utile contribution à une sociologie du sport en plein développement (cf. la récente présentation particulièrement stimulante de J. Defrance, Sociologie du sport, Paris, La découverte, 1995). Il permet de rompre avec les discours de ceux qui ne voient dans l'intérêt pour le football que des engouements futiles. et edérisoires. détournant edes grands problèmes de l'existence individuelle et collective. (p. 5). En fait, comme le dit bien l'auteur, les passions suscitées par le ematch de football. .révèlent brutalement et désignent, grossissent voire anticipent les lignes de force qui traversent le champ social. (ibid.). Cette enquête ethnologique, entreprise au milieu des années quatre-vingt, allie différentes méthodes complémentaires : des entretiens prolongés avec des supporters, des observations dans des stades européens ou ces lieux de discussions interminables que sont les sièges de clubs de supporters et les bars, des analyses de textes mais aussi des enquêtes par questionnaires. Cette recherche se veut également comparative mettant en parallèle le football avec d'autres types de jeux mais surtout le rapport au football dans trois villes de l'Europe du Sud (Naples, Turin et Marseille) ou il suscite un large engouement. C. Bromberger analyse d'abord les processus d'identification à l'objet du spectacle puis les règles de la composition du public et de sa répartition dans le stade. Après s'être intéressé à la rhétorique partisane, il étudie enfin la dimension rituelle du match de football.

À partir de deux matches décisifs pour le titre national (Marseille-Bordeaux et NaplesJuventus de Turin) et d'un derby opposant les deux principales équipes turinoises (le Torino et la Juventus), l'auteur commence à analyser le croisement d'ehistoires de matches, d'shistoires de villes. et d'shistoires de vies. (p. 21 et s.). Là où certains voient des atribus* ou edes hordes de supporters", il montre qu'une passion et une identité (chants, couleurs, notamment) partagées recouvrent une variété d'identifications possibles et plusieurs points de vue sur la vie. (p. 88). Ces matches sont en effet l'occasion de réactiver des oppositions religieuses, politiques, territoriales, etc. Ainsi, l'engouement napolitain et marseillais fournit une revanche symbolique pour deux villes du sud souvent stigmatisées comme le montrent les slogans, les chants ou les banderoles. A travers la composition des tribunes, l'auteur distingue les supporters situées dans les tribunes latérales (est-ouest) qui sont plus âgés et plus -guindés", de ceux, plus jeunes, qui occupent les virages placés derrière les cages des deux équipes (nord-sud). A Marseille, Maître P., avocat d'affaires abonné à la tribune Jean Bouin, voit ainsi dans le football l'occasion de revoir d'anciens camarades de classe, de tisser de nouvelles relations professionnelles et de retrouver sur le terrain ce qui fait de piment de sa vie professionnelle : la joute- (p. 85). Constant P., self-made man, ancien ouvrier chaudronnier, lui aussi habitué de Jean Bouin, est fasciné par la réussite de Tapie et incarne ces supporters soucieux de promouvoir une image moderne de Marseille et de 
sa région. (p. 86). Christian, un chauffeur-livreur en situation précaire âgé de trente ans, offre cun condensé caricatural de traits ordinaires de la pratique du supportérisme. (p. 101). Fréquentant les eUltraso malgré son âge, il joue dans les gradins une carrière de footballeur qu'il n'a pu avoir sur le terrain.

Contrairement aux idées reçues, on ne supporte pas mécaniquement le club de sa ville ou de son entreprise. Dans une seconde partie (-Le football comme métaphore.), C. Bromberger met en évidence la complexité des préférences et des aversions partisanes : elles sont certes liées à des affiliations sociales ou territoriales, dans la mesure où elles exacerbent des conflits existant hors du champ sportif - certaines oppositions peuvent recouvrir des préférences politiques - , mais aussi aux antagonismes propres à l'espace relativement autonome du football (cf. la concurrence, dans les années quatrevingt, entre l'Olympique de Marseille et les Girondins de Bordeaux présidés respectivement par Bernard Tapie et Claude Bez). La variété des possibilités d'identification offerte par le football s'explique par les propriétés même du jeu : il est codifié tout en laissant une grande part à l'aléatoire, il allie division des tâches sur le terrain (postes différents, par exemple) et planification collective (tactique, notamment). Ces identifications multiples sont également rendues possibles par la diversité des postes qui impliquent, moins que dans d'autres sports (basket ou volley, par exemple), des caractéristiques morphologiques spécifiques. Les supporters peuvent aussi s'identifier collectivement au style de jeu d'une équipe, auquel correspond un style de gestion du club et un style de supportérisme, qui portent l'eempreinte de la culture et de l'imaginaire locaux. (p. 130). La composition de l'équipe est le produit à la fois de critères sportifs mais aussi de la recherche d'une équipe idéalisée qui refléterait la population de la ville. Les joueurs, selon leurs caractéristiques spécifiques, sont également l'objet d'identifications différenciées modulées en fonction de l'appartenance sociale, du style de vie et de comportement. (p. 171) : à Marseille, le gardien de but africain Joseph-Antoine Bell est préféré par les jeunes supporters des quartiers nord (dont une forte proportion sont fils d'immigrés) alors que le meneur de jeu, Alain Giresse, plus âgé, est cité par les supporters des classes moyennes et supérieures qui habitent dans les quartiers sud. Pour $\mathrm{C}$. Bromberger, comme le montre l'origine sociale des joueurs, le football n'est donc pas l'apanage exclusif des classes populaires : il reflète ade plus en plus la diversité de la population française: (p. 175).

Cet engouement pour le football ne s'explique pas seulement par la possibilité d'exprimer des identités et des appartenances mais aussi parce qu'il donne à̀ voir, à penser, à commenter toute la palette des attitudes entre lesquelles il faut choisir au fil d'un jour ou d'une vie : l'action solitaire, l'éclat individuel, la coopération, l'abnégation, la ruse, l'affrontement, la temporisation, la prise de risque, la tricherie, etc." (p. 199). Le stade est un lieu où se concrétise l'imaginaire démocratique, exaltant l'égalité des chances, la compétition universelle, le mérite personnel", note encore C. Bromberger (p. 197). Il constitue aussi un creuset de modèles d'organisations sociales. Pour les supporters, le club ou l'équipe sont pensés en référence à la classe d'école, a l'entreprise industrielle ou à la famille. Quant aux arbitres, qui exercent des professions d'autorité (policiers, magistrats, militaires, professeurs...) et aux dirigeants, qui y voient des modèles d'entreprise différents (cf. l'arrivée des emanagers. comme Bernard Tapie ou Jean-Luc Lagardère dans les années quatre-vingt), ils peuvent projeter et mettre en œuvre eleur vision de l'ordre social dans le monde du football. (p. 178).

Dans un troisième temps, l'auteur s'intéresse à la composition du public des stades de football, où s'opère la théâtralisation expressive des appartenances sociales* (p. 205). Prenant l'exemple de l'OM, C. Bromberger note que le public du stade n'est pas exclusivement epopulaire. mais relativement proche de la population de la ville et de sa proche région. A deux spécificités près : il est très majoritairement masculin même si la part des femmes ( $14 \%$ en 1987 ), adolescentes pour la plupart, est en augmentation, et plus juvénile depuis une trentaine d'années ( $83 \%$ des spectateurs ont moins de 39 ans contre 55\% pour la ville de Marseille). Par ailleurs, les employés (31\% du public de l'OM:) sont fortement représentés de même que les Pieds noirs (10\%), les Maghrébins 
issus de la vague migratoire des années soixante (près de $7 \%$ ) et, depuis les années quatre-vingt, les jeunes Beurs qui habitent essentiellement les quartiers nord ( $8 \%$ des moins de dix-sept ans). L'analyse de la répartition du public dans le stade fait apparaître qu'elle ne résulte pas seulement du prix des places mais de la combinaison de variables (âge, profession, quartiers, etc.). Les tribunes se composent de sterritoires. qui renvoient à des appartenances distinctes, chaque egrande étape, biographique se traduisant par sun mode différencié d'occupation de l'espace du stade* (p. 227).

La quatrième partie de l'ouvrage est consacrée à la rhétorique des supporters : le match de football permet l'affirmation de evaleurs" qui s'expriment sous edes formes ritualisées" (p. 263). S'il présente des dimensions guerrières visibles à travers notamment le langage et les vêtements auxquelles se superpose une symbolique du sacrifice, le football permet aussi d'exprimer la virilité (notamment à travers les insultes) ou encore de se glorifier tout en discréditant son adversaire. Le spectacle sportif repose sur une logique partisane equi participe de la nature oppositive de la confrontation", mais aussi sur ale débridement toléré des émotions collectives", comme le notent Norbert Elias et Eric Dunning (Sport et civilisation. La violence maîtrisée, Paris, Fayard, 1994). Une des propriétés spécifiques du football par rapport à d'autres types de spectacles, y compris sportifs, est que le public, dont les pratiques (chants, postures, gestes, etc.) sont très codifiées et ritualisées, est un acteur du match et contribue à daire le spectacle.

Dans la dernière partie, l'auteur analyse les différences et les points communs entre le match de football et le rituel religieux pour conclure à sa uplasticité et [a] sa structure paradoxale - il n'est ni simple spectacle ni rituel reconnu - ; il symbolise sans doute un temps où se brouillent les repères classificatoires des formes de la vie collective. (p. 349). L'ouvrage s'achève sur l'aaffaire OM-VA. où sla métaphore devient et rejoint la réalité. ( $p$. 363) car, selon l'auteur, elle serait révélatrice des problèmes qu'illustre chaque match de football. Elle alimente des discussions sur les "voies du succès dans le monde contemporain permettant de voir les rôles respectifs du emérite, de la chance, de la tricherie, de la justice, (p. 355). "L'affaire. grossit aussi đjusqu'à la démesure, ce qui donne corps à la passion pour le football : la discutabilité, la possibilité d'argumenter et d'interpréter à l'infini. (p. 363). Elle donne enfin à voir l'intensité des phénomènes d'identifications locales et les discussions locales, comme le montre l'analyse du courrier des lecteurs du quotidien Le Provençal.

Pourtant, si l'ouvrage démontre incontestablement l'intérêt de l'approche ethnologique, il en montre également certaines limites. L'auteur aurait pu tirer un meilleur profit d'analyses sociologiques qui ont démontré leur pertinence (cf. le numéro 103 des Actes de la recberche en sciences sociales sur -Les enjeux du football.) et qu'il écarte souvent en les réduisant abusivement (p. 173 et s.). En privilégiant les seules descriptions ethnographiques dans l'enceinte du stade, C. Bromberger n'a sans doute pas assez vu les conditions de production de ce type de spectacle sportif que constituent les matches de football au plus haut niveau. Ce n'est pourtant qu'à condition de rendre compte de l'espace des individus et des groupes qui sont engagés dans le football professionnel italien et français qu'on peut comprendre l'engouement des supporters des trois villes étudiées. On apprend peu par exemple sur la position qu'occupe l'OM par rapport aux clubs dit formateurs. (Nantes, Auxerre, Sochaux...) ou sur les profondes transformations qu'à connu le football professionnel depuis la fin des années soixante-dix, avec notamment l'arrivée massive de capitaux économiques.

Il ne serait pas non plus inutile de montrer comment les médias, et notamment la télévision, participent à la production du match de football jusque dans les tribunes où des références explicites à des propos journalistiques ou à des chaînes de TV figurent sur les banderoles ou font l'objet de slogans. Même s'il est difficile d'en mesurer les effets, les différents types de presse - cf. l'engagement de Canal Plus dans le Paris SG pour concurrencer l'hégémonie de l'OM - contribuent à un engouement *à distance. et leur poids économique est de plus en plus important, comme en témoigne l'accroissement de la part des droits de télévision dans le budget des clubs. C. 
Bromberger, en se concentrant sur une fraction de l'espace du football professionnel en France, ne met qu'en de rares occasions en relation l'espace des sports et les univers politique, économique, juridique, etc. Ses choix de eterrain", des villes ou le football a un quasi monopole des spectacles sportifs importants, lui évitent plus facilement de traiter de la place du football dans l'espace des sports professionnels et, au-delà, dans l'espace des spectacles proposés dans la ville et le pays considéré. Enfin, on aimerait en savoir plus sur les rapports entre le match de football et les compétences spécifiques (connaissances des règles, catégories de perception de l'espace des clubs...) qu'il implique souvent de la part des spectateurs. Mais souligner ces quelques imperfections n'enlève rien au plaisir que le lecteur peut ressentir avec cet ouvrage de C. Bromberger.

Dominique Marchetti

Centre de sociologie de l'éducation et de la culture Ecole des hautes études en sciences sociales

BRUGIDOU (Mathieu), L'élection présidentielle : discours et enjeux polttiques. Une analyse comparée, Paris, L'Harmattan, "Logiques politiques", 1995, 299 pages, bibliographie.

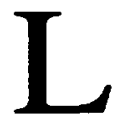

ES ETUDES de lexicologie politique sont depuis quelques années en France en plein renouvellement. Depuis les recherches pionnières de Jean-Marie Cotteret en 1969 sur Le vocabulaire du Général De Gaulle (avec René Moreau, Paris, Presses de la FNSP), l'analyse (statistique) du discours politique a en effet, en particulier grâce aux développements de l'informatique, énormément évolué. Face à une banalisation de ses méthodes liée à une massive importation dans les médias, la lexicologie a connu deux évolutions divergentes. La première est celle d'une technicisation accrue, principalement autour de Maurice Tournier et du laboratoire -Lexicométrie et textes politiques. de l'Ecole normale supérieure de Saint-Cloud. La seconde est celle dont un livre comme celui de $M$. Brugidou est le reflet, c'est-à-dire une lexicologie voulant dépasser le seul cadre d'une lecture interne des discours pour l'intégrer dans une perspective de construction de l'univers symbolique. On peut regrouper actuellement dans ce ccourant. relativement peu important numériquement, né en France avec Jacques Gerstlé et son Langage des socialistes (Paris, Stanké, 1979) outre M. Brugidou, Dominique Labbé, auteur notamment du Vocabulaire de François Mitterrand (Paris, Presses de la FNSP, 1990).

Le grand mérite de ce livre, version remaniée d'une thèse de doctorat soutenue en décembre 1992 à l'Université Paris I sous la direction de J.-M. Cotteret, est de combiner analyse lexicologique et analyse en termes d'enjeu. La perspective proposée en effet, comme l'indique clairement le titre, n'est pas centrée sur un parti ou un acteur politique, comme cela l'a été classiquement dans ce genre d'analyse, mais sur une campagne électorale présidentielle dans sa totalité, en l'occurrence celle de 1988, par ailleurs largement étudiée. On peut penser entre autres à l'ouvrage franco-américain dirigé par L. Kaid, J. Gerstlé et K. Sanders (Mediated Politics in Two Cultures. Présidential Campagning in the United States and France, New York, Praeger, 1991), qui compare les campagnes présidentielles françaises et américaines, ouvrage d'ailleurs curieusement non cité par l'auteur. C'est peut-être aussi l'objet choisi (une campagne électorale) qui a conduit $M$. Brugidou à une plus grande théorisation que dans les analyses lexicologiques classiques. De ce fait, il nous propose dans une première partie intitulée eles enjeux : définitions, une utile clarification de concepts trop couramment utilisés sans explicitations, comme ceux ed'offre politique- et ed'enjeu. d'une part et de "controverse. ou de epriorité. d'autre part. Il se situe par là dans le sillage de l'ouvrage de Daniel Gaxie et Patrick Lehingue sur la constitution des enjeux dans une élection (municipale) : Enjeux municipaux (Paris, PUF, 1984). Parler d'enjeu est en fait insuffisant pour l'auteur, il préfère repérer trois mouvements successifs : priorité, 